\title{
Stabilisation d'une pompe-turbine mono-étage présentant une instabilité en marche à vide
}

\author{
Stabilization of a single-stage turbine-pump presenting an instability \\ when operated at speed-no load
}

par P. Huvet

EDF-CNEH, Le Bourget-du-Lac (F)

\author{
A. J. Engineer, R. N. Pendse, M. V. Brahme \\ Tata Electric Companies, Mumbai (IND) \\ P. K. Dörfler \\ Sulzer Hydro Ltd., Zurich $(\mathrm{CH})$
}

When operated at speed-no load, the vertical single-stage pump-turbine at Bhira pumped storage plant (India) exhibited at rated speed oscillations of speed, head and guide vane opening with a period of 15 seconds. The original PID speed governor could not stabilise this phenomenon. At $105 \%$ of rated speed an additional oscillation with a period of 3.75 seconds was observed. Feedback of the measured penstock pressure in the speed governor improved the stability of speed control at rated speed; but the instability at high speed remained. This problem has been solved by changing the start-up procedure. The turbine inlet valve is opened only partially until synchronisation. The artificial head loss renders the hydraulic condition stable. A series of field tests showed that the novel procedure entails no harmful mechanical side effects.

\section{I INTRODUCTION}

Le comportement de turbines et de pompes-turbines est normalement décrit au moyen d'un ensemble de courbes caractéristiques [1], c'est-à-dire Q11 par rapport à n11 pour le débit et T11 par rapport à n11 pour le couple sur l'arbre'. Ces courbes sont obtenues à partir d'essais en régime permanent sur modèles réduits. A l'exception des effets d'échelle, les courbes du modèle et du prototype sont identiques. Ces courbes dites 4-quadrant jouent un rôle important dans la compréhension et la simulation des phénomènes transitoires qui surviennent dans une centrale.

Dans les courbes 4-quadrant de pompes-turbines monoétages, la condition de marche à vide se trouve normalement au voisinage de la zone dite "S". Dans cette zone, les courbes passent successivement par le nll maximal et minimal, au fur et à mesure que le débit et le couple diminuent (cf. fig. 1). Il a été démontré [3] que, en négligeant les pertes de charge dans la conduite forcée, la limite de la stabilité hydraulique se situe au point maximal de nll.

1 D'autres représentations des caractéristiques d'une pompe-turbine, basées sur la définition de Suter [2], sont également largement employées mais ne seront pas discutées dans cette communication.

\section{DE GROUPE RÉVERSIBLE DE BHIRA}

(cf. également [11] et note à la fin de ce texte)

Un groupe réversible mono-étage a été installé dans la nouvelle centrale de transfert d'énergie par pompage de Bhira dans l'état du Maharashtra (Inde) par Tata Electric Co. La retenue amont est formée par le lac Mulshi, un vaste plan d'eau où le niveau d'exploitation varie entre 590 et $606 \mathrm{~m}$.

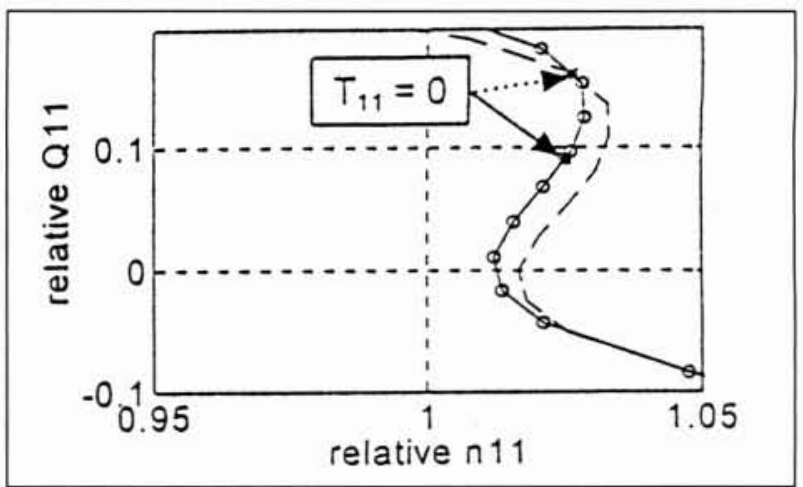

1. Courbes caractéristiques Q11 par rapport à $n 11$ pour un angle des directrices constant de $5^{\circ}$ avec une condition de marche à vide stable (en ligne discontinue) et instable (ligne continue). 
2. Schéma de la STEP de Bhira.

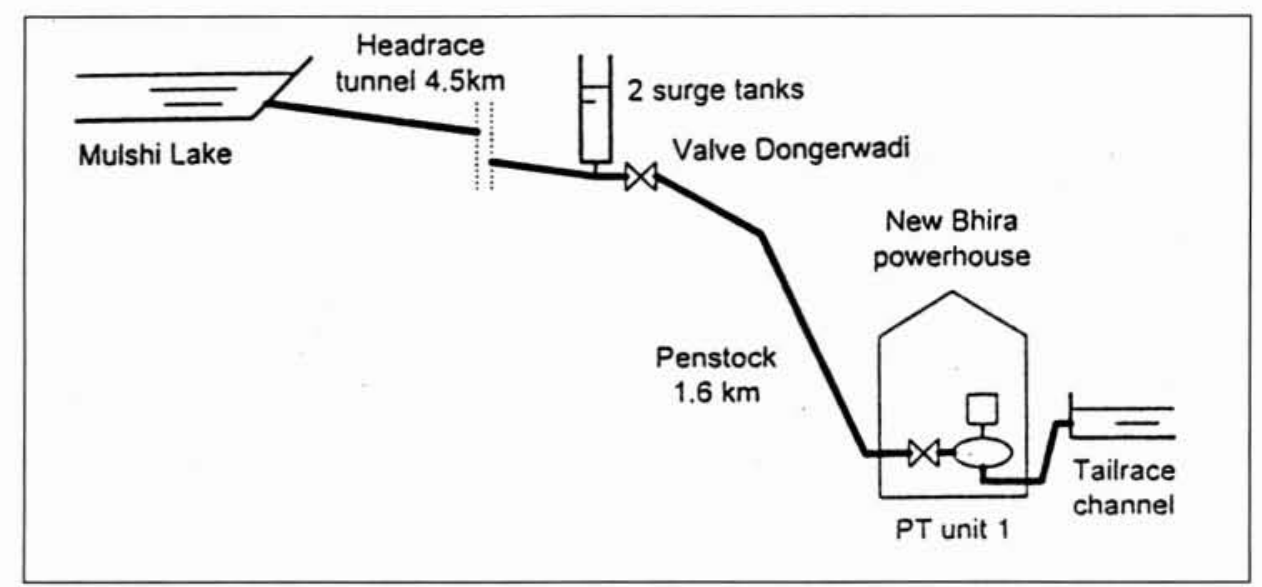

La retenue aval est un petit bassin relié par un canal à écoulement libre à un deuxième bassin de stockage. La nouvelle centrale de Bhira est parallèle à l'ancienne centrale, abritant 6 groupes Pelton, et dispose d'une galerie d'amenée longue de $4,5 \mathrm{~km}$ ainsi que de sa propre conduite forcée, longue de $1,6 \mathrm{~km}$. Une nouvelle cheminée d'équilibre a également été construite. La nouvelle installation a déjà été décrite dans des articles antérieurs [5], [6]. Les tableaux 1 et 2 résument les caractéristiques essentielles de l'usine et du groupe pour la présente communication.

\section{III — PROBLÈMES DE STABILITÉ RENCON- TRÉS PENDANT LA MISE EN SERVICE}

Lorsque le groupe a été amené à fonctionner en marche à vide pour la première fois, le niveau du réservoir amont était près de son maximum. La régulation de vitesse a bien fonctionné près de la limite inférieure de la vitesse contractuelle, mais à vitesse nominale, des oscillations de vitesse, de chute et d'ouverture des directrices se sont manifestées. Ces oscillations étaient faibles mais entretenues (cf. fig. 3 et 9). Le régulateur de vitesse de type PID n'a pas été capable de stabiliser ces oscillations. Il est devenu évident que le groupe était à la limite de la stabilité hydraulique. La période de ces oscillations (de "basse fréquence") était d'environ 15 secondes.

Une évaluation de la formule de C.S. Martin $\left(n^{\circ} 34\right.$ dans [3]) pour la période d'oscillation $T=p\left(2 t_{f} t_{m} / b_{1}\right)^{1 / 2}$ a montré que les oscillations étaient compréhensibles conformé-

Tableau 1 : Caractéristiques dynamiques de la STEP de Bhira

\begin{tabular}{|l|c|}
\hline • Longueur/section galerie d'amenée & $320 \mathrm{~m}^{-1}$ \\
\hline Section totale cheminée d'équilibre & $265 \mathrm{~m}^{2}$ \\
\hline • Longueur/section conduite forcée & $200 \mathrm{~m}^{-1}$ \\
\hline Réflexion d'onde de la conduite forcée & $3,0 \mathrm{~s}$ \\
\hline lère fréquence naturelle conduite forcée & $0,17 \mathrm{~Hz}$ \\
\hline lère fréquence anti-résonance c.f. & $0,30 \mathrm{~Hz}$ \\
\hline • Longueur/section aspirateur + turbine & $10 \mathrm{~m}^{-1}$ \\
\hline Temps de lancer hydraulique & Tw $=1,6 \mathrm{~s}$ \\
\hline
\end{tabular}

ment au modèle de colonne d'eau rigide de Martin. Avec prise en compte des constantes de temps $\left(t_{f}=T_{w}=1,6 \mathrm{~s}\right)$ [4] pour la conduite forcée et pour le groupe $\left(t_{m}=T_{a}=11,7 \mathrm{~s}\right)$, ainsi que du coefficient linéaire de couple relatif basé sur le débit relatif $(b 1=1,33)$, une période de 16,7 secondes a été prévue. L'utilisation du modèle de Martin expliquait bien l'origine des oscillations à basse fréquence sans prendre en compte les variations d'ouverture des directrices. L'amplitude des pressions était de $+/-6 \mathrm{~m} \mathrm{CE}$; la vitesse de rotation variait de $+/-0,25 \%$ et rendait difficile la synchronisation.

A la différence du groupe prototype, le modèle réduit n'avait pas manifesté un comportement instable au point d'emballement. Une partie de cette divergence a été expliquée par un décalage vers le haut de la courbe du couple par rapport à la courbe du débit. Des essais en marche à vide effectués avec les directrices plus ouvertes ont révélé une autre caractéristique dynamique du groupe. Aux ouvertures des directrices au-delà de $26 \%$ et à une vitesse de $105 \%$ de la vitesse nominale, une oscillation quasi-périodique de la vitesse et de la chute avec une période plus courte, de 3,75 secondes, a été observée, illustrée dans la figure 4 .

L'oscillation "moyenne fréquence" a été provoquée par un phénomène d'hystéresis avec une ouverture très large des directrices dans la zone en $\mathrm{S}$. Son amplitude était modeste à l'extrémité aval de la conduite forcée, mais bien supérieure dans les tronçons centraux, puisque la fréquence de $0,27 \mathrm{~Hz}$ était assez proche de la première fréquence d'anti-résonnance $(0,30 \mathrm{~Hz})$.

Tableau 2 : Caractéristiques du groupe en fonctionnement

\begin{tabular}{|l|c|}
\hline Vitesse nominale ${ }^{*}$ ) & $500 \mathrm{tr} / \mathrm{min}$ \\
\hline Puissance nominale ${ }^{*}$ ) & $150 \mathrm{MW}$ \\
\hline Puissance maxi. turbine & $169 \mathrm{MW}$ \\
\hline Débit nominal $^{*}$ ) & $35,17 \mathrm{~m}^{3} / \mathrm{s}$ \\
\hline Chute nominale ${ }^{*}$ ) & $485 \mathrm{~m}$ \\
\hline Chute brute & $500-516 \mathrm{~m}$ \\
\hline Temps de lancer méc. Ta & $11,7 \mathrm{~s}$ \\
\hline Plage de vitesses (turbine) & $97-103 \%$ \\
\hline
\end{tabular}

*) Valeurs spécifiées 


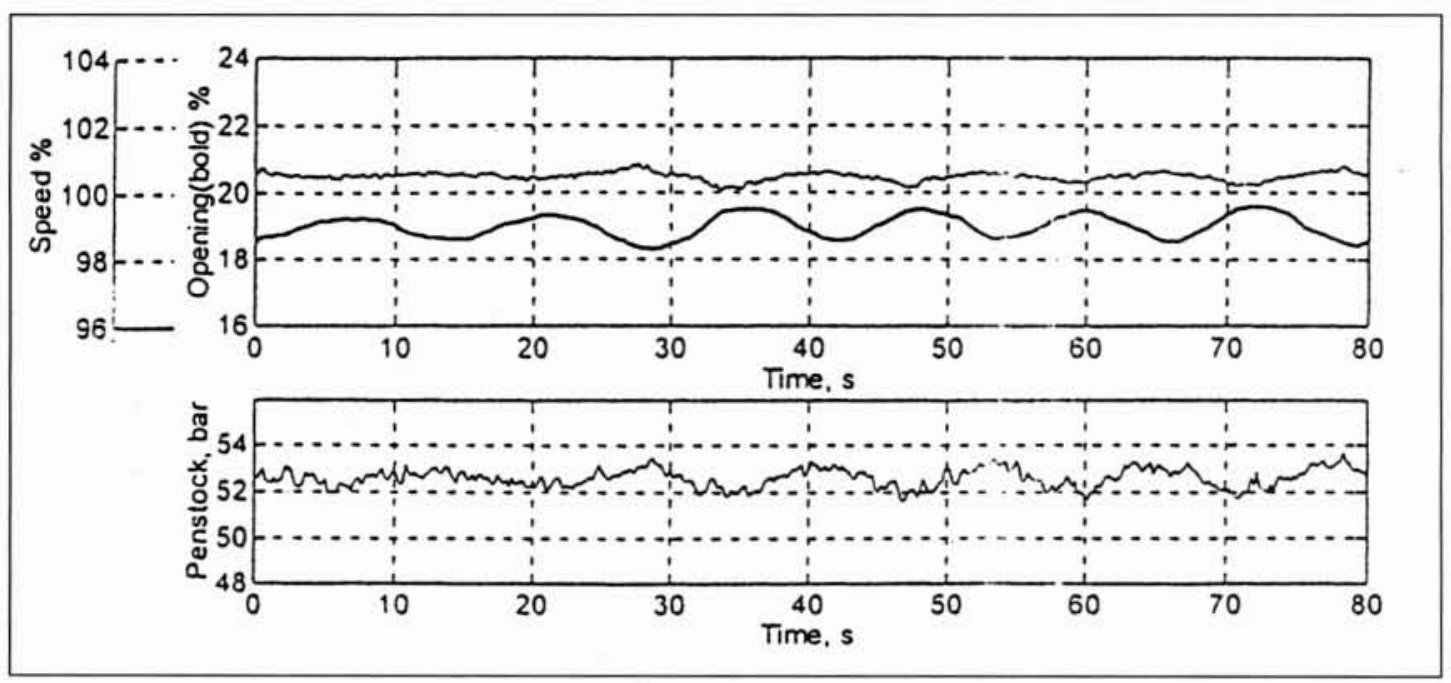

3. Instabilité à "basse fréquence" à une vitesse proche de $100 \%$
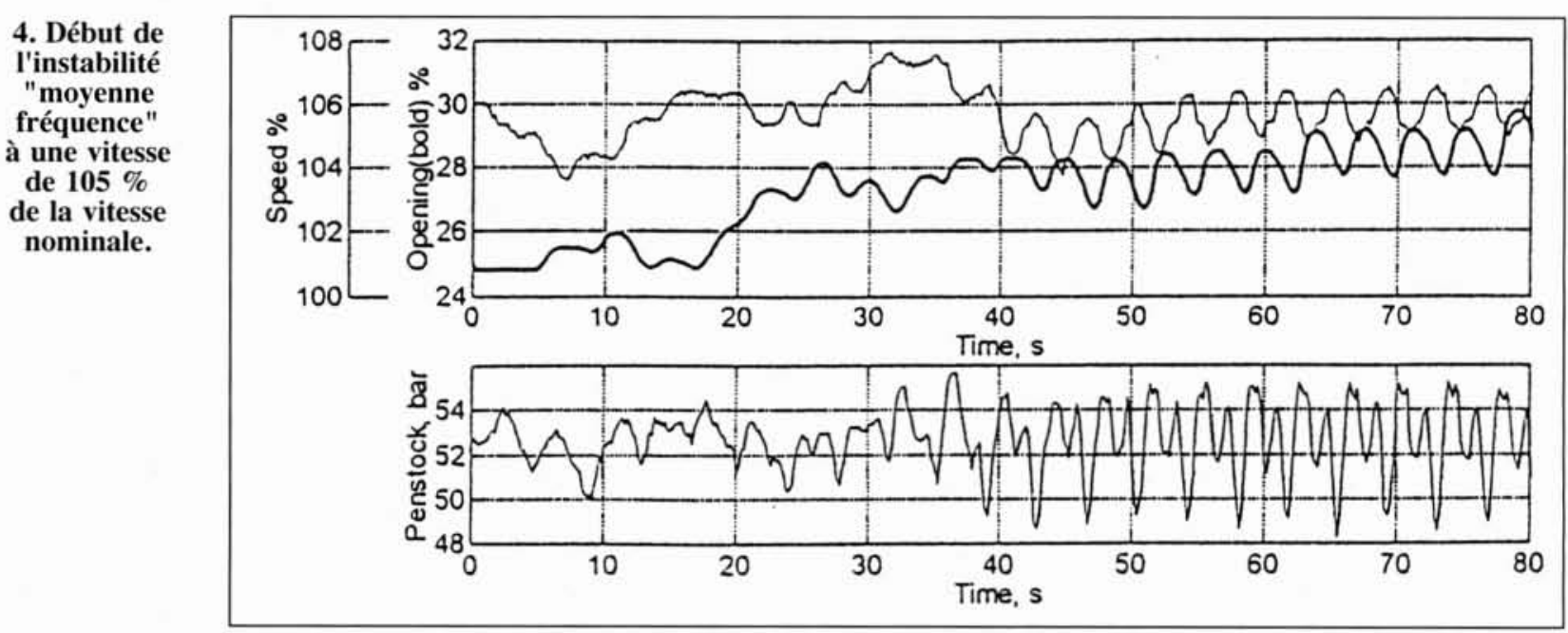

\section{IV — ALGORITHME DE RÉGULATION AMÉLIORÉ}

La pression dans la conduite forcée, telle que mesurée directement à l'amont de la vanne de garde, sert de signal d'entrée à la boucle secondaire dans le régulateur de vitesse. Cette entrée de pression est traitée par une chaîne de filtres (fig. 5) et ajoutée aux données de sortie du régulateur PID. EDF avait recommandé cette adjonction de la pression, sur un principe qui a déjà une longue histoire en France [8]. Par exemple, cette méthode a été utilisée à Montézic, une importante STEP française, et sert également à améliorer la stabilité en cas de synchronisation exceptionnelle à une haute fréquence sur le réseau. EDF a justifié sa proposition au moyen de simulations qui montrent une nette amélioration de la stabilité.

Après mise à jour des courbes des 4 quadrants, pour reproduire les résultats des essais en marche à vide, d'autres simulations de régulation de vitesse ont été menées par Sulzer. Les améliorations prévues ont été confirmées. Les réglages optimaux des paramètres de marche à vide dans le régulateur de vitesse ont été établis par simulations numériques.

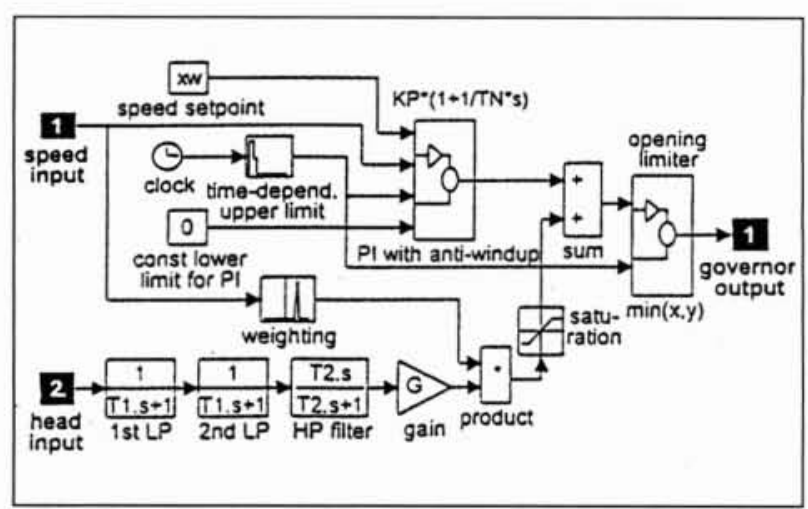

5. Schéma du régulateur de vitesse modifié.

Le régulateur de vitesse modifié a permis une stabilité satisfaisante à la fréquence nominale et à une fréquence légèrement supérieure. Un algorithme de limitation dans le régulateur, agissant sur les consignes de vitesse et de charge, a maintenu le fonctionnement à l'extérieur de la zone "basse chute/haute vitesse" restant provisoirement instable. 


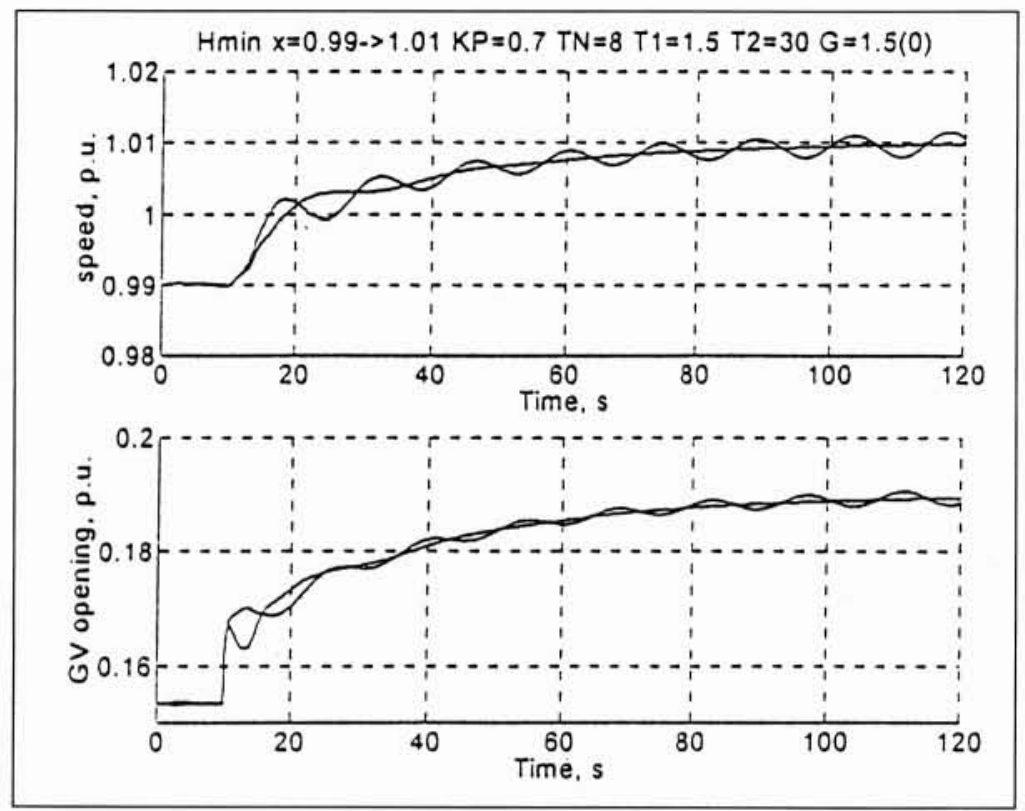

6. Réponse au pas de la consigne de vitesse sans (ligne continue) et avec (ligne discontinue) adjonction de la pression c.f.

mentaires. Dans cette configuration, le démarrage de la turbine et la synchronisation sont effectués avec la vanne sphérique partiellement ouverte $(20 \%)$, un paramètre qui a été déterminé par simulation et optimisé par une série d'essais sur site. La vanne n'est ouverte complètement qu'après la synchronisation. Toutes ces modifications ont été réalisées dans une période de quelques jours, mais uniquement après la démonstration par des essais spécifiques que la procédure modifiée n'entrâ̂nait aucun inconvénient mécanique. La perte de charge requise (14,5 bar dans les cas des phénomènes transitoires les plus extrêmes) représente une fraction de la chute nominale tout à fait acceptable. La structure et les réglages des paramètres du régulateur de vitesse ont pu être conservés.

Puisque la nouvelle utilisation de la vanne sphérique s'écartait en partie de l'utilisation décrite dans les spécifications techniques, il a fallu justifier la mise en œuvre de la nouvelle méthode au moyen d'une série d'essais sur site : - identification du coefficient de débit dans la vanne par rapport à son ouverture (T14),

\section{V $\square$ LA STABILITÉ HYDRAULIQUE : UNE PIERRE D'ACHOPPEMENT}

Si la consigne de vitesse est fixée à ou au-delà de la limite de stabilité hydraulique, même le régulateur modifié ne saurait maintenir la stabilité. Alors que le mode d'oscillation "à basse fréquence" est toujours stabilisé, d'autres modes d'instabilité persistent en l'absence de stabilité hydraulique. Ceux-ci sont liés aux fréquences naturelles de la colonne d'eau élastique dans la conduite forcée. Ainsi, pour assurer un fonctionnement stable, l'instabilité hydraulique doit être supprimée. La littérature [9] montre comment cela peut être réalisé. La méthode consiste en une régulation asynchrone de deux directrices : elle est décrite en détail et a été mise en œuvre avec succès. Par contre, l'impact de cette solution sur le programme de mise en service à Bhira était inacceptable. Il aurait fallu renouveler une partie des essais sur modèle réduit et incorporer des modifications importantes dans l'espace très limité disponible au niveau du mécanisme de manœuvre des directrices.

\section{VI $\square$ PROCÉDURE DE DÉMARRAGE MODIFIÉE}

Une autre solution s'est révélée beaucoup plus simple à mettre en œuvre. Une perte de charge artificielle a été ajoutée à l'extrémité aval de la conduite forcée, en agissant sur la vanne sphérique. Cette technique d'étranglement de la vanne avait déjà été utilisée par Sulzer dans plusieurs centrales afin de contrôler la vitesse de pompes-turbines multi-étages non régulées. A Bhira, la méthode était appliquée à une machine mono-étage régulée pour assurer la stabilité. Si la perte de charge due à la vanne est prise en compte dans les caractéristiques de la pompe-turbine, telle qu'illustrée par la ligne discontinue de la figure 7 , le "groupe modifié" comprenant la vanne et la turbine rend le système hydraulique stable, malgré le fait que la turbine seule (ligne continue) reste instable. La procédure de démarrage a été modifiée et le vérin de la vanne a été équipé d'interrupteurs de fin de course supplé-
- essai de démarrage avec la vanne ouverte à différents degrés (T15),

- essai de régulation de vitesse avec la vanne ouverte à différents degrés (T16),

- essai de synchronisation,

- essai de prise de charge (T17),

- essai de la séquence de démarrage automatique (T19).

Au cours des essais T15 à T17, la turbine et la vanne de garde ont été équipées de capteurs supplémentaires pour mesurer des vibrations éventuelles concernées par l'étranglement partiel. Une attention particulière a été portée à la vanne by-pass et aux fondations, ainsi qu'au couple et aux vibrations des directrices. Un effort a également été fait pour évaluer le risque de cavitation dans la vanne sphérique en régime

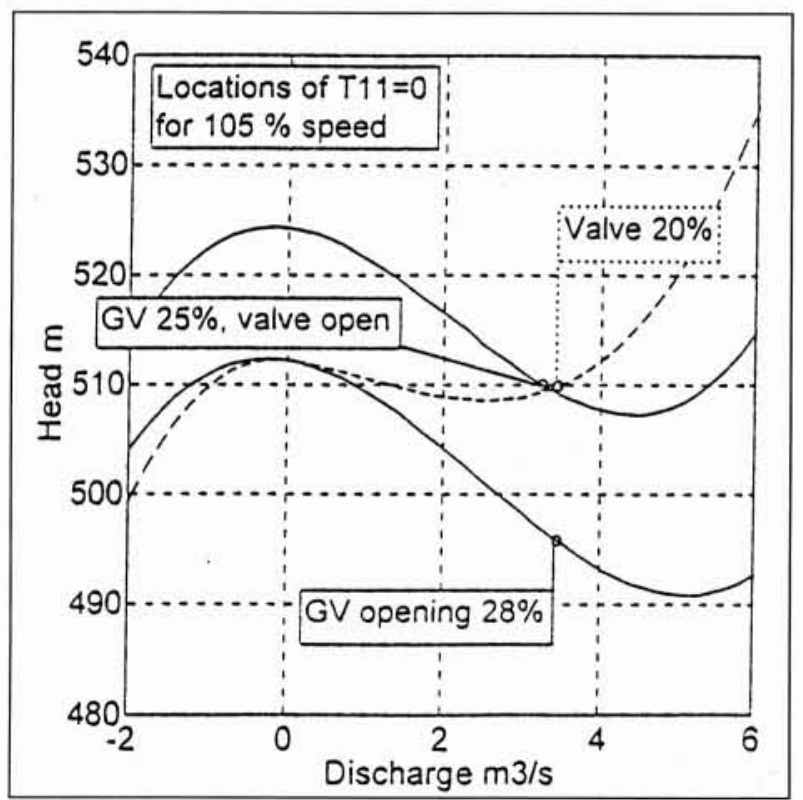

7. Effet stabilisateur de la perte de charge provoquée par une ouverture partielle de la vanne sphérique. 


\begin{tabular}{|l|c|c|c|c|c|c|}
\cline { 4 - 7 } \multicolumn{2}{c}{} & \multicolumn{5}{c|}{ \% ouverture de la vanne } \\
\hline Paramètre & Unité & Limite & $\mathbf{1 0 0}$ & $\mathbf{2 0 , 1}$ & $\mathbf{1 7 , 5}$ & $\mathbf{1 4 , 8}$ \\
\hline Pulsation de pression à l'aval de la vanne & bar p-p & 10 & 1,3 & 2,0 & 2,0 & 2,3 \\
\hline Contrainte au by-pass (amont) & MPa p-p & 26 & 1,3 & 1,6 & 2,0 & 2,0 \\
\hline Contrainte au by-pass (aval) & MPa p-p & 26 & 2,7 & 5,0 & 2,5 & 5,5 \\
\hline Vitesse horiz. des vibrations au pied de la vanne & $\mathrm{mm} / \mathrm{s} 0-\mathrm{p}$ & 25 & 4,0 & 4,8 & 4,5 & 4,5 \\
\hline Vitesse horiz. des vibrations en fondation & $\mathrm{mm} / \mathrm{s} 0-\mathrm{p}$ & 25 & 3,8 & 4,3 & 4,3 & 4,3 \\
\hline Déplacement horiz. dû aux vib. au pied de la vanne & $\mu \mathrm{m}$ p-p & 160 & 9 & 22 & 20 &.- \\
\hline Accélération HF à la manchette de démontage & $\mathrm{m} / \mathrm{s} 2 \mathrm{v} . \mathrm{e}$. & 58 & 54 & 76 & 74 & 64 \\
\hline Couple directrices (le plus défavorable de 2) & $\mathrm{kNm}$ p-p & rel. & 30 & 26 & 28 & 26 \\
\hline Accélération directrices (le plus défavorable de 2) & $\mathrm{m} / \mathrm{s} 2 \mathrm{p}-\mathrm{p}$ & rel. & 240 & 220 & 240 & 220 \\
\hline Vibrations de l'arbre (palier turbine) & $\mu \mathrm{m}$ 0-p & none & 406 & 406 & 375 & 328 \\
\hline
\end{tabular}

Tableau 3. - Quelques résultats des essais de démarrage de la turbine T15.

transitoire, en utilisant la méthode de Gülich [10], basée sur les vibrations transmises à un milieu solide. L'accélération de la manchette de démontage à l'aval de l'obturateur de la vanne a été mesurée avec une largeur de bande de $15 \mathrm{kHz}$.

Les essais $\mathrm{T} 14$ à $\mathrm{T} 16$ ont été renouvelés à différentes positions de l'obturateur de la vanne sphérique. A 17,5 et $20 \%$ de l'ouverture totale de la vanne, l'effet d'étranglement était suffisant pour stabiliser la plage complète des vitesses de fonctionnement, sans force excessive par rapport aux vibrations de la vanne et des directrices. L'ouverture normale au démarrage est de $20 \%$. La limite inférieure, à $17,5 \%$, ne serait atteinte qu'en cas de fuite d'huile du vérin de la vanne au cours des années.

Les critères limitants établis en avance ont été respectés par les essais spécifiques, parfois avec une marge généreuse (tableau 3). Les variations de couple des directrices ont même été réduites par la procédure de démarrage modifiée (fig. 8), La charge réduite dans la turbine compensait les turbulences induites au niveau de l'obturateur de la vanne sphérique.

L'accélération à haute fréquence mesurée à la manchette de démontage à l'aval de la vanne sphérique a été évaluée afin de détecter le risque de cavitation induit par l'étranglement au moyen de l'obturateur de la vanne. Les résultats montrés dans le tableau 3 ne sont pas concluants puisque la valeur de base, avec la vanne ouverte, qui n'est certainement pas sujette à des phénomènes de cavitation, est déjà assez élevée. Le taux de cavitation calculé conformément au concept de Gülich, avec le nombre et la durée réels de démarrage, est de l'ordre de $1 \mu \mathrm{m} / \mathrm{an}$. Aucun contrôle visuel de la manchette n'a été effectué pour le moment.

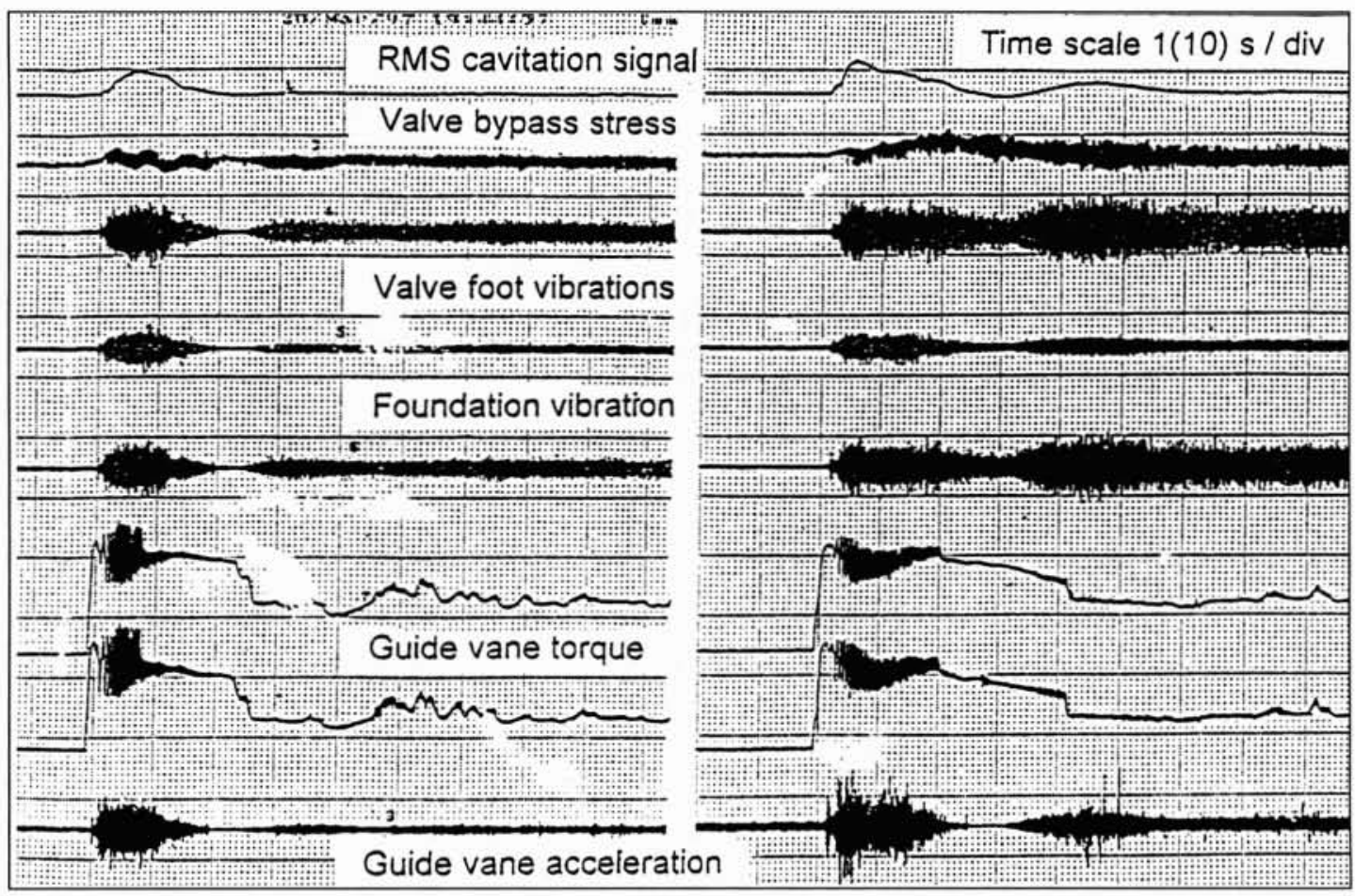

8. Vibrations au cours du démarrage avec la vanne sphérique ouverte complètement (à gauche) et partiellement (à droite) 
9. Amélioration progressive de la stabilité en marche à vide.

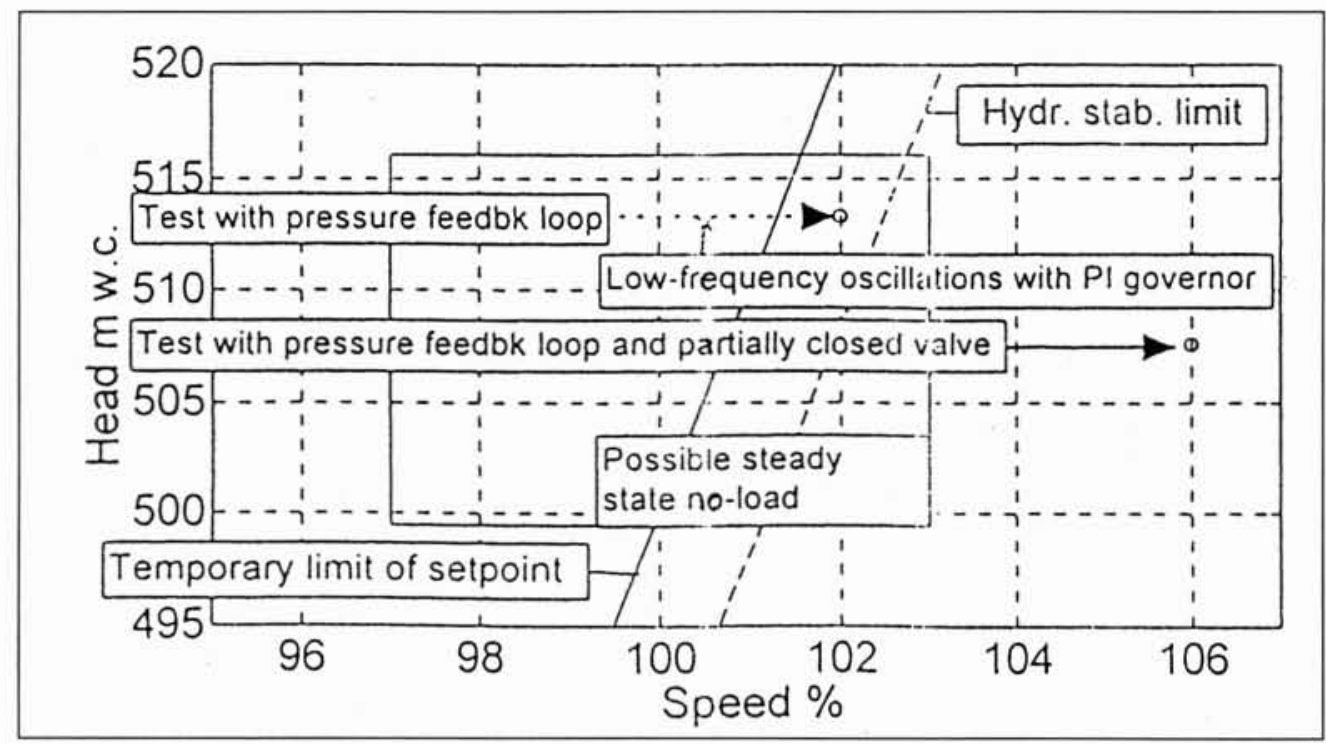

\section{VII — ESSAIS DU RÉGULATEUR DE VITESSE AVEC LA VANNE SPHÉRIQUE PARTIELLEMENT OUVERTE}

Les essais sur le régulateur ont alors été renouvelés avec la vanne sphérique en position partiellement ouverte. Comme avant, les incréments et décréments de la consigne de vitesse ont été testés. Il a été possible de faire fonctionner le groupe à une vitesse jusqu'à $106 \%$ de sa vitesse nominale avec une stabilité satisfaisante. En raison de la perte de charge, les variations de pression et de vitesse dans ces conditions ont été encore plus réduites qu'à la vitesse nominale (fig. 10). Aucune vitesse supérieure n'a été testée. puisque l'essai à $106 \%$ de la vitesse nominale avait déjà pris en compte la fréquence maximale sur le réseau, la chute brute minimale, la baisse de niveau des cheminées d'équilibre, et une marge de sécurité.

\section{VIII $\square$ CONCLUSIONS}

- Le critère de stabilité fourni par [3] est confirmé par l'expérience sur site.

- La simulation des transitoires hydrauliques est un outil précieux pour la résolution de problèmes de régulation.

- Des effets d'échelle importants en marche à vide peuvent se produire dans les courbes 4-quadrant de la pompe-turbine.

- L'adjonction de la pression permet une amélioration significative de la stabilité de la régulation en marche à vide.

- L'ouverture partielle de la vanne de garde est un moyen hydrauliquement efficace de repousser la limite de stabilité en marche à vide et reste mécaniquement tout à fait acceptable.

Echelle de temps $\quad 10 \mathrm{~s} / \mathrm{cm}$

Pression au point haut de la conduite forcée

$2 \mathrm{bar} / \mathrm{cm}$

Ouverture des directrices $\quad 1 \% / \mathrm{cm}$

Vitesse de rotation $\quad 1 \% / \mathrm{cm}$

Pression en pied de la conduite forcée

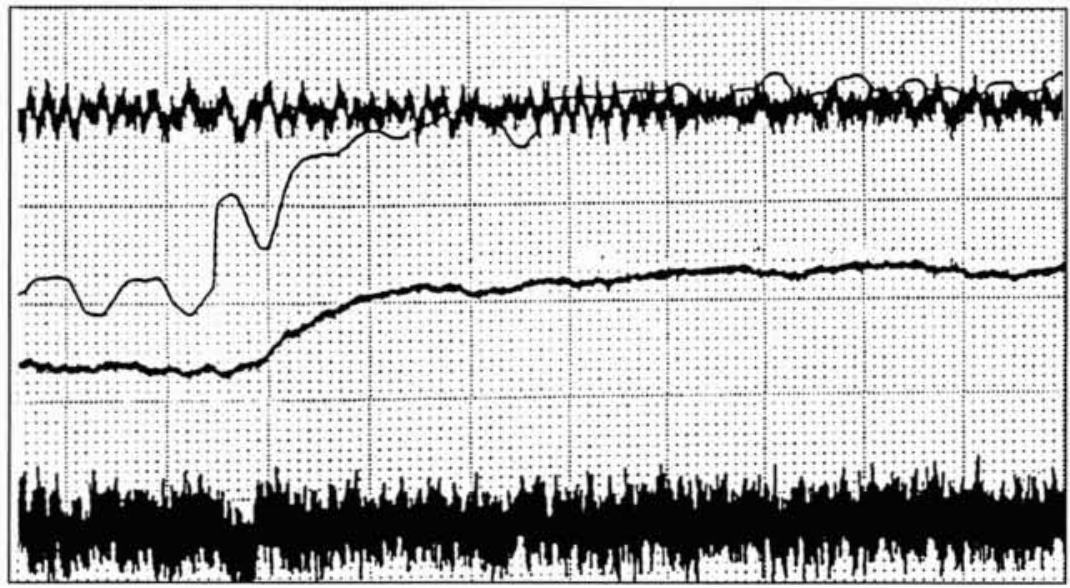

10. Incrément de la consigne de vitesse de $104 \%$ à $105 \%$, avec la vanne sphérique ouverte à $20 \%$. 


\section{pplications industrielles de la mécanique des fluides}

\section{BIBLIOGRAPHIE}

[1]. Warnick, C. C., et al., "Hydropower Engineering", Prentice Hall, 1984, see p. $44 / 45$

[2]. Suter, P., "Representation of pump characteristics for water hammer computations", Techn. Review Sulzer, 1966.

[3]. Martin, C. S., "Stability of pump turbines during transient operation". 5th Internatl. Conf, on Pressure Surges, BHRA, 1986, pp. 61-71.

[4]. IEC publication No, 308, "International code for testing of speed governing systems for hydraulic turbines".

[5]. Bouvier, J. N., Huvet, P., and Truong, Q.M., "Pumped storage for uprating hydro schemes", Water Power \& Dam Construction, Dec. 1992, pp. 38-42.

[6]. Brahme, M. V., Vasudev, S.S., and Ramadas, A., "Suppressing negative water pressures during sudden pump failure", Int. Water Power \& Dam Construction, Jan. '95, pp. 20-25.

[7]. Goede, E., Cuénod, R., Keck, H., and Pestalozzi, J., "3-Dimensional Flow Simulation in a Pump-Turbine", in A.S.M.E. FED - Vol. 86, Industrial and Agricultural Applications of Fluid Mechanics, Editors: T.B. Morrow et al., Book No. H00555 - 1989

[8]. Daniel, J., “Accélération du réglage de vitesse des turbines hydrauliques", La Houille Blanche, 1948, pp. 147-182.

[9]. Klemm, D., "Stabilizing the characteristics of a pump-turbine in the range between turbine part-load and revers pumping operation", Voith Res \&Construction, Vol. 28e (1982).

[10]. Gülich, J. F., "Diagnosis of Cavitation in Centrifugal Pumps", Sulzer Techn. Rev., 1/1992
[111. Jaquet, M., Engineer, A. J., Pendse, R. N., Brahme, M. V., and Bouvier, J.-N., "Bhira - The first pump-turbine in private utility in India". Paper presented at the International Conference on Accelerated hydropower development and pumped storage in India, Dec. 1-3, 1997, New Delhi. India.

\section{Informations complémentaires sur le projet}

Pour faire face à la demande de production en période de pointe et satisfaire la fourniture d'énergie pendant la nuit, Tata Electric Co. (TEC) a engagé la réalisation d'une station de transfert d'énergie par pompage. Bhira a été choisi en raison de l'existence des retenues supérieure et inférieure. Tata Consulting Engineers (TCE) a effectué l'étude de faisabilité du projet. La Banque Mondiale a soutenu le projet financièrement. TCE a servi de maître d'œuvre pour le projet, Dr. C.S. Martin a étudié les transitoires hydrauliques, et EDF a servi d'ingénieur conseil pour la revue des équipements électromécaniques. Les prestations d'EDF ont été prolongées jusqu'à la phase des essais et de la mise en service. Un groupement d'entreprises piloté par Sulzer-Siemens a été engagé pour la fourniture, le montage, les essais et la mise en service de l'alternateur-moteur, de la pompe-turbine, de la vanne sphérique et des matériels électromécaniques associés. Bhira est la première STEP construite pour une société électrique privée en Inde. 